\title{
Pharmacokinetic-based failure of a detergent virucidal for SARS-COV-2 nasal infections
}

Charles R. Esther Jr.

University of North Carolina at Chapel Hill

Kyle S. Kimura

Vanderbilt University Medical Center

Yu Mikami

University of North Carolina at Chapel Hill

Caitlin E. Edwards

University of North Carolina at Chapel Hill

Suman R. Das

Vanderbilt University Medical Center

Michael H. Freeman

Vanderbilt University Medical Center

Britton A. Strickland

Vanderbilt University Medical Center

Hunter M. Brown

Vanderbilt University Medical Center

Bronson C. Wessinger

Vanderbilt University Medical Center

Veerain C. Gupta

Vanderbilt University Medical Center

Kate Von Wahlde

Vanderbilt University Medical Center

Quanhu Sheng

Vanderbilt University Medical Center

Li Ching Huang

Vanderbilt University Medical Center

Daniel R. Bacon

University of North Carolina at Chapel Hill

Adam J. Kimple

University of North Carolina at Chapel Hill

Agathe S. Ceppe

University of North Carolina at Chapel Hill

Takafumi Kato 
University of North Carolina at Chapel Hill

Raymond J. Pickles

University of North Carolina at Chapel Hill

Scott H. Randell

University of North Carolina at Chapel Hill

Ralph S. Baric

University of North Carolina at Chapel Hill

Justin H. Turner

Vanderbilt University Medical Center

Richard C. Boucher ( $\nabla$ richard_boucher@med.unc.edu )

University of North Carolina at Chapel Hill

\section{Research Article}

Keywords: SARS-CoV-2, topical virucidal agent, irrigation

Posted Date: May 14th, 2021

DOI: https://doi.org/10.21203/rs.3.rs-500168/v1

License: (c) (1) This work is licensed under a Creative Commons Attribution 4.0 International License. Read Full License 


\section{Abstract}

The nose is the portal for SARS-CoV-2 infection, suggesting the nose as a target for topical antiviral therapies. Because detergents are virucidal, Johnson and Johnson's Baby Shampoo (J\&J) was tested as a topical virucidal agent in SARS-CoV-2 infected subjects. Twice daily irrigation of J\&J in hypertonic saline, hypertonic saline alone, or no intervention were compared $(n=24 /$ group). Despite demonstrated safety and robust efficacy in in vitro virucidal assays, J\&J irrigations had no impact on viral titers or symptom scores in treated subjects relative to controls. Similar findings were observed administering J\&J to infected cultured human airway epithelia using protocols mimicking the clinical trial regimen. Additional studies of cultured human nasal epithelia demonstrated that lack of efficacy reflected pharmacokinetic failure, with the most virucidal J\&J detergent components rapidly absorbed from nasal surfaces. This study emphasizes the need to assess the pharmacokinetic characteristics of virucidal agents on airway surfaces to guide clinical trials.

\section{Introduction}

The nose is the primary portal for infection by SARS-CoV- $2^{1,2}$. A fraction of inhaled SARS-CoV-2 impacts on nasal surfaces, which provide an ACE2 receptor-rich environment that promotes robust viral proliferation ${ }^{1}$. From nasal surfaces, SARS-CoV-2 can spread locally to the olfactory region and more widely to the oral cavity and lung ${ }^{1,3}$. Accordingly, nasal protection represents an important strategy to limit SARS-CoV-2 infection and transmission ${ }^{4}$. In addition to physical means, e.g., masks, topical administration of a spectrum of virucidal agents to nasal surfaces has been advocated for this purpose, including iodine, metal, and detergent-based molecular entities ${ }^{5-7}$.

Otolaryngologists utilize high-volume normal or hypertonic saline (HTS) rinses to cleanse the nasal cavity. A common agent added to saline rinses to provide gentle detergent activity is Johnson and Johnson's (J\&J) Baby Shampoo ${ }^{8}$. The J\&J Baby Shampoo/saline (J\&J/S) combination, typically $1 / 2$ to 1 tsp/240 $\mathrm{ml}$ saline ( $8 \mathrm{oz}$ ), is safe and effective for treatment of chronic bacterial infections of the nasal cavity ${ }^{9}$. Because detergents are virucidal for enveloped viruses, including SARS-CoV-2, we tested J\&J/S as a topical virucidal for the treatment of SARS-CoV-2 nasal cavity infections ${ }^{5,10,11}$.

\section{Results}

The first component of this study investigated the in vitro safety and virucidal activity of clinically utilized dilutions of $J \& J / S^{9}$. Due to the urgency to limit SARS-CoV-2 nasal infections during the ongoing pandemic, a clinical trial of the safety and efficacy of $\mathrm{J} \& \mathrm{~J} / \mathrm{S}$ high-volume rinses in subjects with nasal SARS-CoV-2 cavity infections was then performed in parallel with the pharmacokinetic (PK) and virucidal pharmacodynamic (PD) studies of J\&J/S in SARS-CoV-2-infected cultured human nasal epithelia (HNE).

Safety studies revealed that exposure of HNE cultures to serial dilutions of J\&J/S induced transient decreases in barrier function (transepithelial resistance) at concentrations above 1/2 tsp J\&J/240 ml 
saline without changes in cell composition (Fig. S1A, B). Assays of virucidal activity demonstrated that non-toxic concentrations of J\&J/S ( $1 / 2 \mathrm{tsp} \mathrm{J} \& \mathrm{~J} / 240 \mathrm{ml}$ saline) were virucidal in vitro against NL63 coronavirus and respiratory syncytial virus (Fig. S1C, D). Notably, rapid and robust SARS-CoV-2 virucidal activity was observed when SARS-CoV-2 virus at varying titers was exposed to this concentration of J\&J/S (Fig. 1A).

Having demonstrated in vitro safety and virucidal activity, an outpatient clinical trial of J\&J/HTS nasal irrigation was performed in subjects with nasal swab qPCR-documented SARS-CoV-2 infection. Seventytwo subjects were randomized into three groups: 1 ) twice daily nasal irrigation with $1 / 2$ tsp J\&J Baby Shampoo in $240 \mathrm{ml} \mathrm{HTS}$; 2) twice daily irrigation with HTS alone; or 3) no intervention (see Table 1, Fig. S1E). The primary study endpoint was qPCR-measured SARS-CoV-2 nasal viral load $4 \mathrm{~h}$ after irrigation over the three-week study interval. Other endpoints included patient-reported symptom scores assessed using a modified Wisconsin Upper Respiratory Symptom-21 Survey (WURSS-21) and daily temperatures.

No reductions in qPCR-measured nasal cavity viral load were observed in the intervention groups compared to control group (Fig. 1B, Fig. S1F). Similarly, no significant improvements in nasal symptoms were reported in either intervention group compared to the control group (Fig. 1C). No safety signals were observed, e.g., no changes in WURSS-21 smell indices were observed in the irrigation groups to indicate irrigation-mediated spread of SARS-CoV-2 to olfactory epithelia (Fig. S1G), or daily temperature changes (not shown).

Insights into the disparity between the virucidal activity of J\&J/S in vitro (Fig. 1A) and the absence of clinical effectiveness (Fig. 1B) emerged from the PK/PD studies of J\&J/S in HNE cultures. For these studies, HNE cultures were inoculated at $t=0$ with D614G SARS-CoV-2 at an MOI of $0.1^{12}$. Samples for viral qPCR quantitation, utilizing the SARS-CoV-2 nucleoprotein (N1) primers employed in the clinical study, and viral titers were obtained via PBS lavage of HNE culture surfaces $48 \mathrm{~h}$ post inoculation (pi) ${ }^{13}$. At $72 \mathrm{~h} \mathrm{pi,} \mathrm{J \& J/S} \mathrm{or} \mathrm{PBS} \mathrm{lavages} \mathrm{were} \mathrm{administered} \mathrm{to} \mathrm{HNE} \mathrm{culture} \mathrm{surfaces,} \mathrm{fluids} \mathrm{aspirated} 10$ min later, and titering performed. Note, viral qPCR was not technically accurate in virus-inactivated J\&J Baby Shampoo lavage solutions. Importantly, the in vitro J\&J/S lavage volume-to-HNE surface area ratio (200 $\mu \mathrm{l} / \mathrm{cm}^{2}$ ) approximated the ratio in vivo, i.e., $240 \mathrm{ml}$ lavage spread over $150 \mathrm{~cm}^{2}$ nasal surface area ${ }^{14}$, and the $10 \mathrm{~min}$ in vitro J\&J/S lavage residence time mimicked the in vivo contact time of lavages with nasal surfaces $^{8,15}$. Viral qPCR and titering assays were performed on PBS lavages obtained $4 \mathrm{~h}$ after the $72 \mathrm{~h}$ $\mathrm{J} \& \mathrm{~J} / \mathrm{S}$ or PBS lavage, i.e., at $76 \mathrm{~h} \mathrm{pi}$, and the next day at $96 \mathrm{~h}$ pi.

Like previous data ${ }^{1}$, PBS lavage samples revealed productive SARS-CoV-2 infection of HNE at $48 \mathrm{~h}$ pi (Fig. 1D, E), with little change in viral titer at $72 \mathrm{~h} \mathrm{pi} \mathrm{(Fig.} \mathrm{1E,} \mathrm{Fig.} \mathrm{S1I).} \mathrm{Surprisingly,} \mathrm{the} \mathrm{titers} \mathrm{measured} \mathrm{in}$ the $\mathrm{J} \& \mathrm{~J} / \mathrm{S}$ lavage at $72 \mathrm{~h}$ pi were also not significantly different from those measured at $48 \mathrm{~h}$ in these samples (Fig. 1E, Fig. S1I, J). Furthermore, no differences in qPCR-measured viral load or viral titers from $48 \mathrm{~h}$ values were observed in the J\&J/S or PBS lavaged HNE cultures over the 76-96 h pi interval (Fig. 1D, E, Fig. S1H, I). 
Collectively, these data demonstrate that J\&J/S exhibits virucidal activity against SARS-CoV-2 in a test tube in vitro (Fig. 1A) but not when administered onto nasal surfaces in vitro or in vivo (Fig. 1B, D, E). What is noteworthy about SARS-CoV-2-infected nasal surfaces is the continual production and release of SARS-CoV-2 virus onto nasal surfaces over time (Fig. 1E) ${ }^{1}$. Accordingly, a virucidal agent must continually remain on nasal surfaces to produce durable and clinically meaningful virucidal activity in the nasal cavity. However, it is difficult to maintain effective concentrations of topical agents on nasal surfaces for two reasons. First, nasal surfaces are protected by a mucociliary transport system that clears nasal surfaces of topically deposited agents within $10 \mathrm{~min}^{8,15,16}$. Second, the nasal surfaces exhibit rapid transepithelial absorption of topically applied agents, a property accessed for drug delivery ${ }^{17-19}$.

We, therefore, studied the retention of topically applied $\mathrm{J} \& \mathrm{~J} / \mathrm{S}$ on nasal surfaces. J\&J Baby Shampoo is a proprietary mix of agents. Mass spectrometric analysis of $\mathrm{J} \& \mathrm{~J} / \mathrm{S}$ indicated that the second listed agent after water, and dominant detergent, is cocamidopropyl betaine (CAPB) of varying chain lengths (Fig. $\mathrm{S} 1 \mathrm{~K})$. Mass spectrometric analysis of PBS lavages obtained $1 \mathrm{~min}$ or $30 \mathrm{~min}$ after administration of $\mathrm{J} \& \mathrm{~J} / \mathrm{S}$ to HNE surfaces revealed that the longer chain CAPB components were absorbed from HNE surfaces within $1 \mathrm{~min}$ of administration (Fig. 1F). These data suggest that the failure of J\&J/S to reduce SARS-CoV-2 titer in the HNE studies (Fig. 1D, E) reflected the rapid transnasal absorption of the longer chain, more active virucidal components of J\&J/S from the lavage fluid (Fig. S1L) ${ }^{11,20}$.

\section{Discussion}

Our data do not support use of $\mathrm{J} \& \mathrm{~J} / \mathrm{S}$ as a topical virucidal agent for treatment of active SARS-CoV-2 nasal infections. The failure of J\&J/S to treat SARS-CoV-2 did not reflect a defect in the intrinsic virucidal activity of this agent. Rather, the result reflected a PK failure to maintain for prolonged intervals the concentrations of J\&J virucidal ingredients on nasal surfaces required to inactivate SARS-CoV-2 virions continuously being shed onto nasal surfaces. SARS-CoV-2-infected nasal surfaces continually produce and release SARS-CoV-2 virus onto nasal surfaces over time (Fig. 1E) ${ }^{1}$. Accordingly, a virucidal agent must continually remain on nasal surfaces to produce durable and clinically meaningful virucidal activity in the nasal cavity. However, it is difficult to maintain effective concentrations of topical agents on nasal surfaces for two reasons. First, nasal surfaces are protected by a mucociliary transport system that clears nasal surfaces of topically deposited agents within $10 \mathrm{~min}^{8,15,16}$. Second, the nasal surfaces exhibit rapid transepithelial absorption of topically applied agents, a property accessed for drug delivery ${ }^{17-19}$.

These data suggest that the failure of $\mathrm{J} \& \mathrm{~J} / \mathrm{S}$ to reduce SARS-CoV-2 titer in the HNE studies reflected primarily the rapid transnasal absorption of the longer chain, more active virucidal components of J\&J/S from the lavage fluid. Longer chain detergents have greater antiviral activity but also readily absorb onto cell surfaces ${ }^{11,20}$, which we show limits their potential as antiviral agents. Although our studies focused on CAPB as the dominant detergent in J\&J, similar findings likely apply to similar detergents in J\&J or other products. We also cannot rule out the possibility that mucociliary transport may have also contributed to the lack of efficacy in the clinical trial. 
This study emphasizes the need to assess a priori the PK characteristics of virucidal agents considered for topical use against SARS-CoV-2 nasal cavity infections. Cell culture systems such as the ones utilized in our study can be utilized to rapidly evaluation topical antiviral agents to determine which retain their efficacy when applied to human airway epithelia, particularly when exposure times are limited to mimic the impact of mucociliary clearance. Such studies can be utilized to inform clinical trials for SARS-CoV-2 and other respiratory viral infections.

\section{Methods}

Methods are described briefly below, with further information in the supplement.

\section{In Vitro Safety, Pharmacodynamic, and Pharmacokinetic Studies}

\section{Safety Studies in Airway Epithelia:}

Apical surfaces of well-differentiated air-liquid interface human nasal epithelial (HNE) cultures were exposed to $5 \mu \mathrm{L}$ of various dilutions of $\mathrm{J} \& \mathrm{~J} / \mathrm{S}$ for $5 \mathrm{~min}$, washed with $600 \mu \mathrm{L}$ of cell culture media ${ }^{1}$, and transepithelial resistance $\left(R_{t}\right)$ measured utilizing an EVOM (World Precision Instruments). Parallel cultures after J\&J/S exposure were fixed in $4 \%$ PFA and processed for whole mount histologic analyses ${ }^{1}$.

\section{Virucidal Assays in Vero and Cultured Human Nasal Cells:}

SARS-CoV-2 D614G virus stocks at $10^{7} \mathrm{PFU} / \mathrm{ml}$ were incubated with $\mathrm{J} \& \mathrm{~J}$ at 1:1 or 1:100 dilutions for 90 min at $37^{\circ} \mathrm{C}$, then serially diluted in PBS for plaque assay on Vero cells ${ }^{1}$. Virucidal activity of J\&J Baby Shampoo was also assessed in recombinant NL63-coronavirus (NL63-CoV) ${ }^{21}$ and Respiratory Syncytial Virus (RSV) ${ }^{22}$ expressing GFP. $100 \mathrm{ml}$ of NL63 or RSV were mixed with PBS or J\&J/S, incubated at $37^{\circ} \mathrm{C}$ for $5 \mathrm{~min}$ or $90 \mathrm{~min}$, then virus titers determined by Median Tissue Culture Infectious Dose (TCID50/ml) on Vero cells.

Well differentiated HNE cultures were inoculated with $200 \mu$ of SARS-CoV-2 D614G at an MOI of 0.1, incubated at $37^{\circ} \mathrm{C}$ for $90 \mathrm{~min}$, following which the inoculum was removed and cultures lavaged $2 \mathrm{X}$ with $500 \mu \mathrm{l} \mathrm{PBS}$ to remove residual virus. Apical lavages were performed with $200 \mu \mathrm{l}$ PBS for $10 \mathrm{~min}$ at $37^{\circ} \mathrm{C}$ at $48 \mathrm{hpi}, 76 \mathrm{hpi}$, and $96 \mathrm{hpi}$ as described in the text. HNE were also lavaged with a J\&J/S or PBS at 72 hpi. Apical lavages were stored at $-80^{\circ} \mathrm{C}$ until analyzed by qPCR to measure viral load and plaque assay to determine viral titer.

Individual surfactant components of CAPB including octanoylamide propylbetaine (C8 chain length), lauroylamide propylbetaine (C12 chain length), and palmitoylamide propylbetaine (C18 chain length) were obtained from Toronto Research Chemicals (Toronto, CA). SARS-CoV-2 D614G virus stock at $10^{7}$ 
$\mathrm{PFU} / \mathrm{ml}$ was incubated with each surfactant individually at $1 \mathrm{mg} / \mathrm{mL}, 0.1 \mathrm{mg} / \mathrm{mL}$, or $0.01 \mathrm{mg} / \mathrm{mL}$ for 90 min at $37^{\circ} \mathrm{C}$. Titers of active virus post treatment were determined by the plaque titer assay as described above.

qPCR Quantitation of SARS-CoV-2 Viral Load from HNE Cultures: Apical culture lavages were inactivated with urea, and RNA was isolated using Direct-zol RNA Kits (\#R2073, ZYMO RESEARCH). Briefly, $100 \mu \mathrm{L}$ apical lavage was mixed with $300 \mu \mathrm{L}$ TRI reagent followed by $400 \mu \mathrm{L} 99 \%$ ethanol, transferred to the spin column, centrifuged with wash buffer then eluted with $50 \mu \mathrm{L}$ RNase-free water. Total RNA was reversed transcribed into cDNA with iScript Reverse Transcription Supermix for RT-qPCR (\#1708841, Bio-Rad, CA, USA) and virus copy number was quantitated using the SARS-CoV-2 (2019-nCoV) CDC qPCR Probe Assay kit (\#10006770, Integrated DNA Technologies).

LC-MS/MS: Five $\mu \mathrm{L}$ of a 1:100 dilution of J\&J Baby Shampoo was analyzed using chromatographic and mass spectrometric conditions as previously described ${ }^{23}$. Full scans were run in positive mode with electrospray interface (ESI).

Statistical Analyses of in vitro studies: For transepithelial airway culture resistance, the Dunnet test (control $=$ PBS) was utilized to assess differences in $R_{t}$ as a function of $J \& J / S$ concentrations at serial time points. To analyze the effects of J\&J/S vs. PBS on magnitudes of SARS-CoV-2 infection of HNE, the changes from $48 \mathrm{~h}$ at each successive time point were analyzed with a repeated measure model. Interaction of time and treatment (J\&J/S vs PBS) was explored and $48 \mathrm{~h}$ values were included as covariates. Post-hoc comparisons of treatments at each time point were adjusted with Holm. P values $<0.05$ were considered significant. Changes in viral titers with J\&J or CAPB lipids were assessed using one sample T-tests against expected titers, with Bonferroni corrections to account for multiple testing.

\section{Clinical Trial of J\&J Baby Shampoo}

Study Population and Enrollment: The clinical study was approved by the Vanderbilt University Medical Center Institutional Review Board and Biosafety Committee and registered on clinicaltrials.gov (NCT 04347538). A CONSORT diagram for the study is shown in Fig. S1E. Patients with a positive qualitative qRT-PCR test for the SARS-CoV-2 virus were randomized to one of three treatment groups: 1) no intervention, 2) hypertonic nasal saline irrigations BID, and 3) hypertonic nasal saline irrigations with $1 / 2$ teaspoon surfactant (Johnson's Baby Shampoo; Johnson \& Johnson Inc.; New Brunswick, NJ) BID. Hypertonic saline solution consisted of $240 \mathrm{~mL}$ of distilled water with 2 packets of NeilMed brand buffered salt (NeilMed Pharmaceuticals; Santa Rosa, CA). Nasal lavage was performed in each nostril 
using NeilMed brand Sinus Rinse bottles. Randomization, enrollment, and registration took place via REDCap (Vanderbilt University, Nashville TN). Further data, including statistical analyses, are in the supplement.

\section{Declarations}

\section{Acknowledgments}

We thank Eric C. Roe for editorial assistance. We also thank all the subjects who participated in the study, as well as study personnel. This work was supported by National Institutes of Health grants UH3 HL123645, R01 HL136961, P30 DK 065988, and P01 HL108808 (RCB); P30-ES10126 (CRE); R21Al142321-01A1S1, R21Al142321 (JHT); and U01 Al151797 and R01 Al108197 (RSB); and by Cystic Fibrosis Foundation grants BOUCHE19R0 and BOUCHE19XXO (RCB); and MIKAMI19XXO (YM). This project was also supported by the North Carolina Policy Collaboratory at the University of North Carolina at Chapel Hill with funding from the North Carolina Coronavirus Relief Fund established and appropriated by the North Carolina General Assembly. The project was also supported by the National Center for Advancing Translational Sciences, National Institutes of Health, through Grant KL2TR002490 (AJK). The content is solely the responsibility of the authors and does not necessarily represent the official views of the $\mathrm{NIH}$. Authors have no conflicts of interest to report.

\section{Author Contributions}

CRE wrote the manuscript and performed mass spectrometric analysis. KSK, YM, TK, SHR performed cell culture experiments. CEE performed experiments with SARS-CoV-2, and RJP performed experiments with NL63 and RSV. SRD, MHF, BAS, HMB, BCW, VCG, KVW, QS, LCH, and DRB performed the clinical trial and analysis with input from AJK. ASC provided biostatistical support. RSB, JHT, and RCB provided overall oversight of the study.

\section{Additional Information}

The authors have no competing interests to report.

\section{References}

1. Hou, Y. J. et al. SARS-CoV-2 Reverse Genetics Reveals a Variable Infection Gradient in the Respiratory Tract. Cell 182, 429-446 e414, doi:10.1016/j.cell.2020.05.042 (2020).

2. Sanche, S. et al. High Contagiousness and Rapid Spread of Severe Acute Respiratory Syndrome Coronavirus 2. Emerg Infect Dis. 26, 1470-1477 https://doi.org/10.3201/eid2607.200282 (2020). 
3. Huang, N. et al. Integrated single-cell atlases reveal an oral SARS-CoV-2 infection and transmission axis.Nat Med(2021 (in press)).

4. Ziegler, C. G. K. et al. Impaired local intrinsic immunity to SARS-CoV-2 infection in severe COVID-19. bioRxiv. https://doi.org/10.1101/2021.02.20.431155 (2021).

5. Parhar, H. S. et al. Topical preparations to reduce SARS-CoV-2 aerosolization in head and neck mucosal surgery. Head Neck. 42, 1268-1272 https://doi.org/10.1002/hed.26200 (2020).

6. Frank, S. et al. In Vitro Efficacy of a Povidone-lodine Nasal Antiseptic for Rapid Inactivation of SARSCoV-2. JAMA Otolaryngol Head Neck Surg. 146, 1-5 https://doi.org/10.1001/jamaoto.2020.3053 (2020).

7. Farrell, N. F., Klatt-Cromwell, C. \& Schneider, J. S. Benefits and Safety of Nasal Saline Irrigations in a Pandemic-Washing COVID-19 Away. JAMA Otolaryngol Head Neck Surg. 146, 787-788 https://doi.org/10.1001/jamaoto.2020.1622 (2020).

8. Isaacs, S., Fakhri, S., Luong, A., Whited, C. \& Citardi, M. J. The effect of dilute baby shampoo on nasal mucociliary clearance in healthy subjects. Am J Rhinol Allergy. 25, e27-29 https://doi.org/10.2500/ajra.2011.25.3583 (2011).

9. Chiu, A. G. et al. Baby shampoo nasal irrigations for the symptomatic post-functional endoscopic sinus surgery patient. Am J Rhinol. 22, 34-37 https://doi.org/10.2500/ajr.2008.22.3122 (2008).

10. Jahromi, R., Mogharab, V., Jahromi, H. \& Avazpour, A. Synergistic effects of anionic surfactants on coronavirus (SARS-CoV-2) virucidal efficiency of sanitizing fluids to fight COVID-19. Food Chem Toxicol. 145, 111702 https://doi.org/10.1016/j.fct.2020.111702 (2020).

11. Kracht, M. et al. Antiviral and hemolytic activities of surfactin isoforms and their methyl ester derivatives. J Antibiot (Tokyo). 52, 613-619 https://doi.org/10.7164/antibiotics.52.613 (1999).

12. Hou, Y. J. et al. SARS-CoV-2 D614G variant exhibits efficient replication ex vivo and transmission in vivo. Science. 370, 1464-1468 https://doi.org/10.1126/science.abe8499 (2020).

13. Rosas-Salazar, C. et al. SARS-CoV-2 Infection and Viral Load are Associated with the Upper Respiratory Tract Microbiome. The Journal of allergy and clinical immunology. https://doi.org/10.1016/j.jaci.2021.02.001 (2021).

14. Gizurarson, S. The relevance of nasal physiology to the design of drug absorption studies. Adv Drug Deliv Rev. 11, 329-347 (1993).

15. Pandya, V. K. \& Tiwari, R. S. Nasal mucociliary clearance in health and disease. Indian J Otolaryngol Head Neck Surg. 58, 332-334 https://doi.org/10.1007/bf03049581 (2006).

16. Black, A. et al. Impairment of nasal mucociliary clearance in woodworkers in the furniture industry. $\mathrm{Br}$ J Ind Med. 31, 10-17 https://doi.org/10.1136/oem.31.1.10 (1974).

17. Rygg, A. \& Longest, P. W. Absorption and Clearance of Pharmaceutical Aerosols in the Human Nose: Development of a CFD Model. J Aerosol Med Pulm Drug Deliv. 29, 416-431 https://doi.org/10.1089/jamp.2015.1252 (2016). 
18. Türker, S., Onur, E. \& Ozer, Y. Nasal route and drug delivery systems. Pharm World Sci. 26, 137-142 https://doi.org/10.1023/b:phar.0000026823.82950.ff (2004).

19. Shang, Y. et al. Prediction of nasal spray drug absorption influenced by mucociliary clearance. PLoS One. 16, e0246007 https://doi.org/10.1371/journal.pone.0246007 (2021).

20. Takai, E., Hirano, A. \& Shiraki, K. Effects of alkyl chain length of gallate on self-association and membrane binding. J Biochem. 150, 165-171 https://doi.org/10.1093/jb/mvr048 (2011).

21. Donaldson, E. F. et al. Systematic assembly of a full-length infectious clone of human coronavirus NL63. J Virol. 82, 11948-11957 https://doi.org/10.1128/JVI.01804-08 (2008).

22. Zhang, L., Peeples, M. E., Boucher, R. C., Collins, P. L. \& Pickles, R. J. Respiratory syncytial virus infection of human airway epithelial cells is polarized, specific to ciliated cells, and without obvious cytopathology. J Virol. 76, 5654-5666 (2002).

23. Esther, C. R. Jr. et al. Sialic acid-to-urea ratio as a measure of airway surface hydration. Am J Physiol Lung Cell Mol Physiol. 312, L398-L404 https://doi.org/10.1152/ajplung.00398.2016 (2017).

\section{Tables}

Table 1. Clinical and demographic characteristics of study participants.

\begin{tabular}{|c|c|c|c|c|}
\hline & $\begin{array}{c}\text { No Intervention } \\
(n=24)\end{array}$ & $\begin{array}{l}\text { Hypertonic Saline } \\
(\mathrm{n}=24)\end{array}$ & $\begin{array}{c}\text { Saline + Surfactant } \\
(\mathrm{n}=24)\end{array}$ & $\begin{array}{c}\mathbf{p} \\
\text { value }\end{array}$ \\
\hline Age & $39 \pm 15$ & $39 \pm 15$ & $44 \pm 18$ & 0.68 \\
\hline Sex, no. male (\%) & $14(58)$ & $12(50)$ & $9(38)$ & 0.35 \\
\hline BMI & $29.6 \pm 7.2$ & $28.5 \pm 6.3$ & $28.9 \pm 5.9$ & 0.89 \\
\hline $\begin{array}{l}\text { Symptomatic days before } \\
\text { diagnosis }\end{array}$ & $2.0(1.0-3.0)$ & $2.5(1.0-4.8)$ & $2.0(1.0-3.5)$ & 0.79 \\
\hline Smoking, no. (\%) & $1(4)$ & $2(8)$ & $2(8)$ & 0.81 \\
\hline \multicolumn{5}{|l|}{ Comorbidities } \\
\hline Diabetes, no. (\%) & $3(12)$ & $0(0)$ & $3(12)$ & 0.2 \\
\hline Heart disease, no. (\%) & $2(8)$ & $0(0)$ & $1(4)$ & 0.18 \\
\hline Hypertension, no. (\%) & $4(17)$ & $4(17)$ & $6(25)$ & 0.7 \\
\hline $\begin{array}{l}\text { Chronic lung disease, no. } \\
\text { (\%) }\end{array}$ & $4(17)$ & $3(12)$ & $2(8)$ & 0.68 \\
\hline
\end{tabular}

Categorical data presented as number with percentages. Continuous data presented as medians with interquartile range or means \pm standard deviation.

\section{Figures}



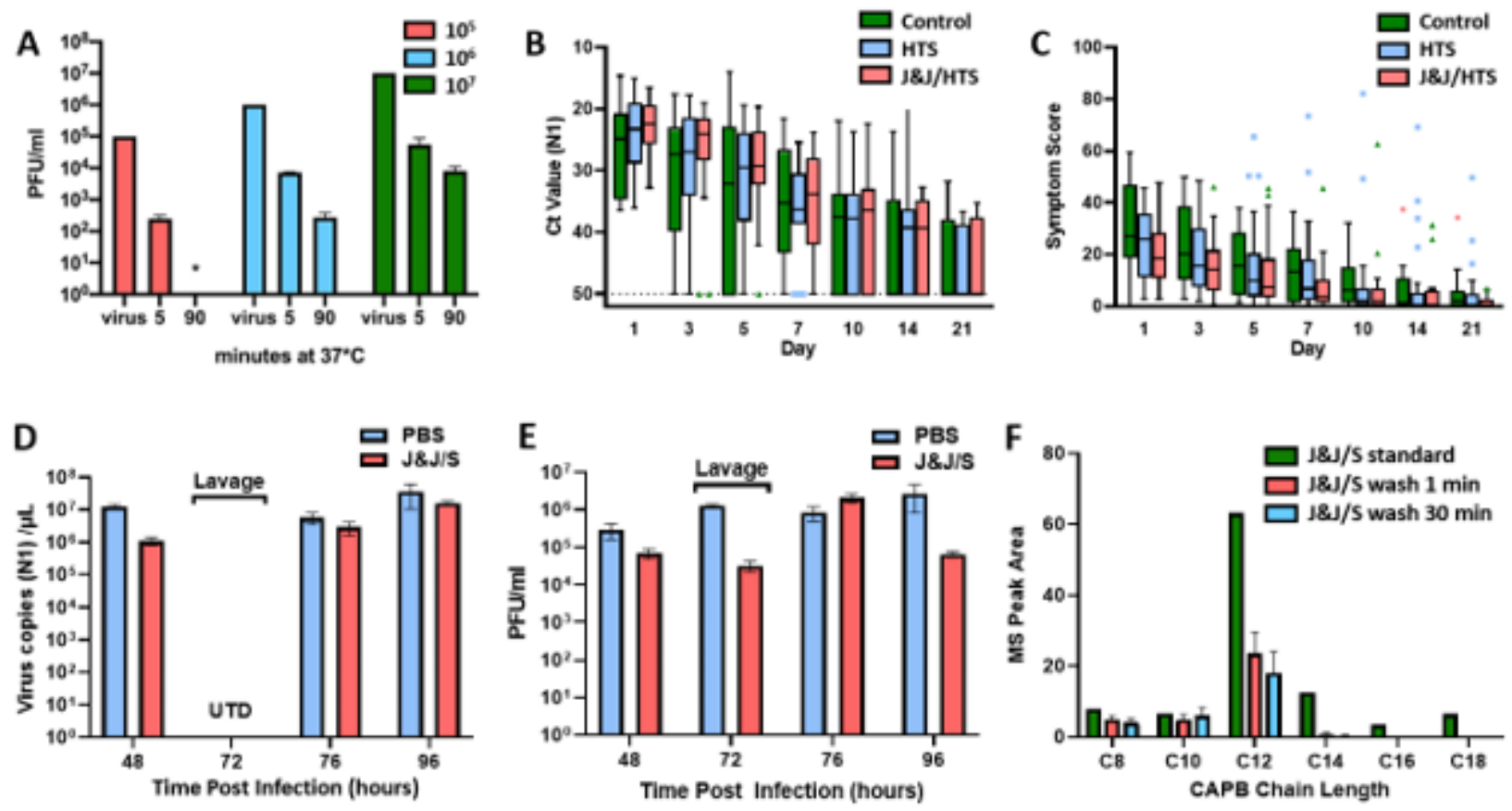

Figure 1

Preclinical, clinical, and pharmacokinetic data. Panel A depicts virucidal activity of J\&J Shampoo (1/2 tsp $\mathrm{J} \& \mathrm{~J} / 240 \mathrm{ml}$ saline) at a 1:1 dilution with SARS-CoV-2 viral stocks assayed varying viral titers. Initial SARS-CoV-2 stock titers ranged from 105-107 PFU/ml. * $=p<0.05$ vs. starting viral titer; ND=zero titer detected. Panel B depicts cross point (Ct) PCR-based measure of N1 primer-based viral load in the nasal cavity of SARS-CoV-2-infected subjects as a function of treatment group. $\mathrm{N}=72 ; 24 /$ group. Note, lower absolute $\mathrm{Ct}$ value reflects greater viral load. Panel $\mathrm{C}$ depicts nasal WURSS-21 symptom score of SARSCoV-2-infected subjects as a function of treatment group over the study interval. Panel D depicts the nucleocapsid gene region 1 (N1) qPCR-based measure of SARS-CoV-2 viral copies in human nasal epithelial culture lavages collected at the designated times post D614G SARS-CoV-2 inoculation. Note, interference of the J\&J Shampoo and the sample viral deactivation (8 M urea) protocol caused technical interference that obviated measurements of the $72 \mathrm{~h}$ pi J\&J/S samples. Statistical analyses of changes for J\&J/S and PBS lavage groups from $48 \mathrm{~h}$ pi values shown in Fig. S1H. Panel E depicts D614G SARSCoV-2 viral titer data at the times post inoculum designated. Statistical analyses of changes in J\&J/S and PBS 72 lavage groups compared to $48 \mathrm{~h}$ pi values shown in Fig. S1I. Panel F depicts results of mass spectroscopic analyses of application of J\&J Shampoo (200 $\mu \mathrm{l}, 1 / 2 \mathrm{tsp} / 240 \mathrm{ml}$ normal saline concentration) to cultured HNE and harvested $1 \mathrm{~min}$ and 30 min later for analysis. Cocamidopropyl betaine (CAPB) length denoted.

\section{Supplementary Files}

This is a list of supplementary files associated with this preprint. Click to download. 
- JJPaperSuppSR042621.docx 